\title{
Survey on PDL: Personal Digital Locker
}

\author{
Tejas Patil ${ }^{1}$, Gaurav Kulkarni Rajat ${ }^{1}$, Apoorva Golhar ${ }^{1}$, Anand Mane ${ }^{1}$, Prof. Rachana Sable ${ }^{2}$ \\ Bachelors in Engineering, Computer, G.H Raisoni Institute of Engg and Technology, Pune, India ${ }^{1}$ \\ Professor, Computer, G.H Raisoni Institute of Engg and Technology, Pune, India ${ }^{2}$
}

\begin{abstract}
The distinctive ID system provides distinctive identification numbers to Indian residents. UID variety which might not simply help the govt. to trace down people, however would create life so much easier for voters as they'd not ought to submit multiple documents on every occasion they require to avail a brand new public, private and government service. This method can contain personal details like name, sex, address, legal status, photo, identification mark and fingerprint biometric. The unique identification number are within the fashioned of twelve digit alphanumeric variety to supply a lot of security. UID system helps to manage persons wants in his/her generation by employing a single UID is employed as license number, Voter ID card variety, identification number in any organization, bank account variety, personal or skilled details. This system can produce government jobs for pc sector within the development, implementation and maintenance of the UID system.
\end{abstract}

Keywords: Anonymity, attribute-based encryption, Digital Locker.

\section{INTRODUCTION}

Currently, in India, almost all of the government issued documents and documents related to health are in physical form across the country. This means every time a resident needs to share the document with any requestor to available any service, an attested photo copy or copy of any healthcare report either in physical form or on scanned form is available. Use of physical copies of documents creates huge problem in terms of manually verification, paper storage, manual arrangement, etc. which creates high cost and inconvenience. This creates problem for various requestors to verify the authenticity of these documents, thus, creating ambiguity for usage of fake documents/certificates also.

Due to the nature of these documents not having a strong authenticity attached to it, anyone with same name can misuse someone else's document in anyway. Remote data Authenticity checking is very important in cloud storage. It can make the clients verify data which comes from external source whether data is kept in without downloading the whole data. In some applications case, the clients have to store their data on multi-cloud servers. At the same time, the authenticity checking protocol is very important and efficient in order to save the verifier's cost. we propose a remote data authenticity checking model in multi-cloud storage which checks integrity of personal documents. The formal system model and security related model are given. Based on the bilinear pairings, a concrete protocol is designed. The proposed protocol is provably secure under the hard hardness assumption of the standard CDH problem. In addition to the structural advantage of elimination of certificate management, our system purposed protocol is also efficient and flexible. Based on the client's authorization, the proposed protocol can realize private verification, delegated verification, and public verification.

\section{REVIEW OF RELATED LITERATURE}

In survey we tend to determined user comment is extremely vital AADHAAR assurance of individualism and centralized online biometric identification would be the premise of building multiple services and applications.

- It will substitute all different identification problems as well as illegal migration, in banking and money transactions, fraud, health connected matters, in education sector, welfare sector, within the election method, observation economical law and order.

-it's simple to count country's population with none mistakes and take a action to regulate population that gradually increase in excess quantity.

- It'll offer support in providing correct identification to the people and this UID are going to be joined with a person's Passport range, driving licence, PAN card, Bank Accounts, elector ID etc. and every one this data are going to be checked by info

\section{III.RESERCH METHODOLOGY}

The UIDM system is to develop Unique-id management that creates distinctive positive identification to specific subject of country. further as management of perspective knowledge, information of that subject. Secondary goal is that we offer some feature during this project that ar attempt to facilitate individuals to achieve/reduces their stress of traditional life.

Basically, the UIDM system that is handled by moderator, consistent with recent work the "Aadhaar" system that is developed for providing Unique-id. This method not provides completely different feature. This method may be accessed by the subject, and different individuals 
Vol. 5, Issue 12, December 2016

associated with governmental activity, therefore we have a tendency to attempt to achieve straightforward interface, and user friendly system.

\section{IV.SYSTEM ARCHITECTURE}

\section{A. Design and Architecture}

We describe the algorithmic rule that is employed to elucidate however the system goes to figure, i.e. the method logic behind it, the flow diagram that represents the picturing of the process logic and eventually the information flow chart (Context Level) of the UIDM system.

\section{B. Algorithm}

Security mechanism in UID project - if there's no physical Identity card or electronic open-end credit, then however can UID system validate its voters.

For implementing this, two different processes got to followed, the primary one being the recording method and therefore the other - the authentication process.

\section{Registration Process:}

In the 1st method, the UIDM builds up a centralized database consisting of UID, biometric record and numerous other details of the person. The UIDM allocates a singular twelve digit alpha numeric range (UID) that is haphazardly generated by the most pc to each subject.

Then a biometric information record is created by scanning the ten fingerprints of an individual. This biometric information is labeled to the person's unique twelve digit range (UID). The UID labeled to the biometric record of a subject is later employed in the authentication process.

\section{Authentication Process:}

In the second method, whenever someone has be known whether he/she could be a real one, a contemporary biometric scan is made then the scanned image is shipped to the centralized server.

The server takes the contemporary scanned biometric image as an input and compares it with all the already keep biometric records within the information. If a relevant match found is found, then the person is selected to be a real subject.

\section{Flow Chart of System}

Regarding the flow of the system. It'll not show the operations performed very well however simply access the operation and wherever it goes once the operation has been performed.

It shows the various conditions like ("if else") if one condition isn't true than wherever the flow can come and from where can it begin once more or wherever the flow can terminate once.

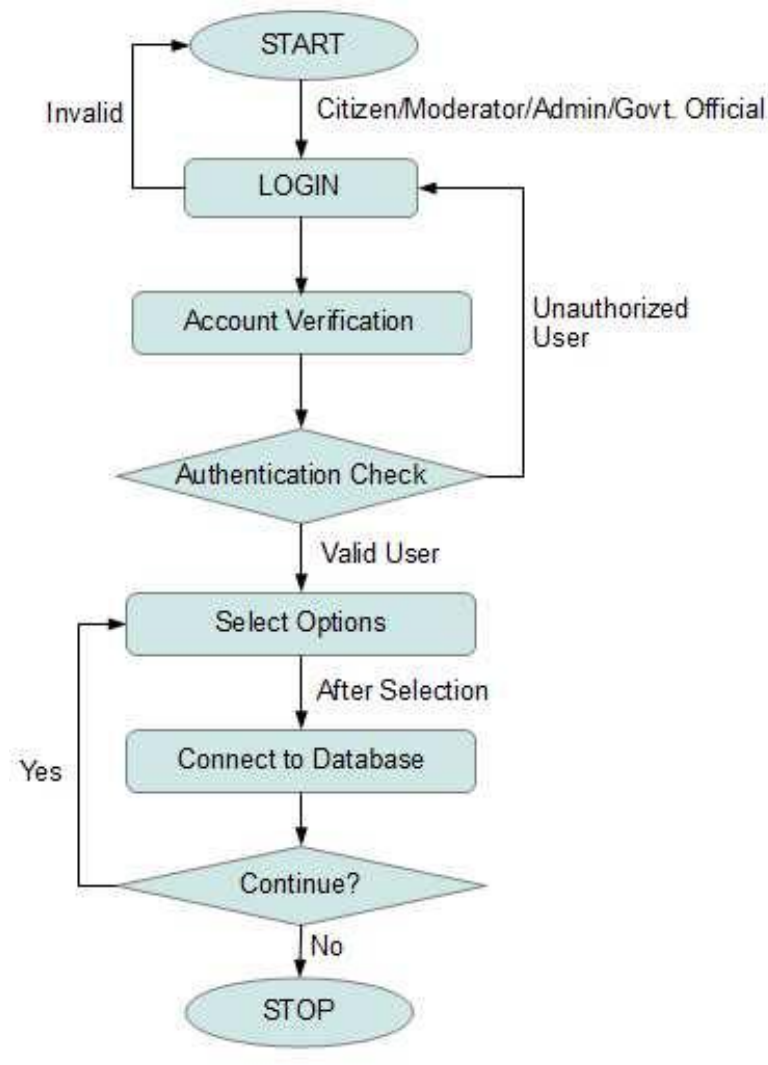

Fig.1. Flow chart

\section{Data Flow Diagram}

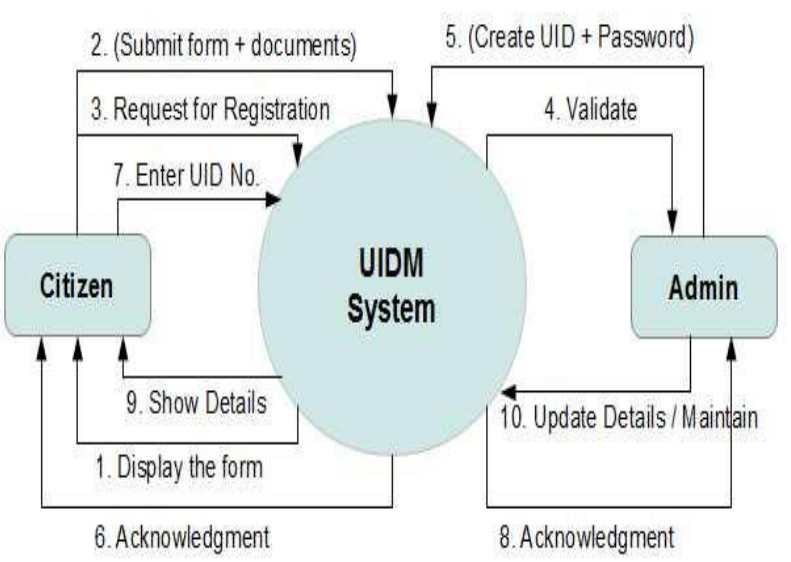

Fig 2. Data Flow Diagram

\section{DISCUSSION}

A single distinctive range is employed thus decreasing manual labour and increasing potency as each detail is available on the only click and reducing the efforts in maintaining completely different ID databases. The UID can cut back the duplication, an endeavour to create faux documents. The purpose of this UID system is to supply one distinctive number to any or all the voters to extend the protection and verification method by introducing the Biometric authentication technology, and therefore characteristic contraband immigrants and terrorists. 


\section{VI.CONCLUSION}

Personal Digital Document Locker will be good initiative for safe custody of the important documents such as PAN card; passports etc. which determines the nationality of the Citizens of India electronically. Moreover other important information will also be saved on to the card. This would in turn result in much more transparency, authenticity and eradication of corruption to the maximum extent possible. This is a refreshing move towards making 'a digitized economy' and thereby ensuring broadband connectivity in the rural areas. Thus, for the program to be a success, it becomes imperative that there is absolute coordination between the departments and utmost commitment on the part of ministries

\section{ACKNOWLEDGMENT}

We thank our partners, who provided insight and expertise that greatly assisted the research. We thank our Prof. Rachana Sable madam for inspiring us to do this project and her comments on the manuscript.

\section{REFERENCES}

[1] 'Unique Identifiers: a brief introduction' by Brian Green and Mark Bide (February 1999)

[2] 'Unique Identification Number: Panacea for India's Problems?' by Harsh Varshan Pant, Nihar Ranjan Pande, Jeetendra Pande published in International Journal of Technology and Applied Science, Vol. 2, pp.12- 15, 2011 ISSN: 2230-9004

[3] 'Unique Identification System' by Alankrit Patnaik and Deepak Gupta published in International Journal of Computer Applications (0975 - 8887) Volume 7- No.5, September 2010

[4] 'Unique Identification Number And E-governance Security' by Subash Chander published in International Journal of Computing and Business Research (IJCBR) Volume 1, N. 1 December - 2010. 